\title{
Chronic thromboembolic pulmonary hypertension: a distinct disease entity
}

Irene Lang

Affiliation: Internal Medicine II, Division of Cardiology, Medical University of Vienna, Vienna, Austria.

Correspondence: Irene Lang, Medical University of Vienna, Internal Medicine II, Division of Cardiology, Waehringer, Geurtel, 18-20, 1090 Vienna, Austria. E-mail: irene.langdameduniwien.ac.at

ABSTRACT Chronic thromboembolic pulmonary hypertension (CTEPH) is a distinct subtype of pulmonary hypertension $(\mathrm{PH})$. One disease hypothesis is that CTEPH results from the non-resolution of venous thromboembolism. CTEPH is characterised by the presence of obstructive fibrotic thromboembolic material in the major pulmonary vessels, with concomitant microvascular arteriopathy, resulting in progressive $\mathrm{PH}$. The clinical presentation of CTEPH is similar to pulmonary arterial hypertension with nonspecific symptoms, but it is distinguished from pulmonary arterial hypertension by the presence of mismatched segmental defects on the ventilation/perfusion scan. The exact prevalence and incidence of CTEPH are unknown, but are thought to have been underestimated in the past. CTEPH is unique among the subgroups of $\mathrm{PH}$ in that it is potentially curable with pulmonary endarterectomy, a surgical intervention intended to remove the occlusive material from the pulmonary vasculature. However, in some patients the obstructions are technically inaccessible or the risk/benefit ratios are unfavourable, making the condition inoperable. It is thought that the involvement of the smaller, more distal vessels is a target for medical treatment. Untreated, CTEPH may result in right heart failure and death. The pathophysiological mechanisms which cause CTEPH are complex and have not yet been fully elucidated.

0 @ERSpublications

CTEPH is distinct from other types of pulmonary hypertension, both in terms of its pathophysiology and treatment http://ow.ly/L54ag

\section{Introduction}

Chronic thromboembolic pulmonary hypertension $(\mathrm{CTEPH})$ is a distinct form of pulmonary hypertension $(\mathrm{PH})$ characterised by mechanical obstruction of the pulmonary arteries, which is caused by the presence of organised fibrotic thrombi tightly attached to the medial layer of the elastic pulmonary arteries, replacing the normal intima [1]. This thromboembolic material may completely occlude the lumen of the affected artery, with associated pitting or roughening of the intimal surface, formation of bands and webs traversing the vascular lumen and partial recanalisation [2]. The consequences of this pulmonary artery occlusion are an increase in pulmonary vascular resistance with subsequent progressive $\mathrm{PH}$ and eventual right heart failure, which may be fatal $[3,4]$.

Although the pathogenesis of CTEPH is yet to be fully elucidated, it has long been understood that it arises as a complication of acute pulmonary embolism (PE) subsequent to venous thromboembolism (VTE) [2]. The pathophysiological mechanisms that prevent complete resolution of the embolic material in CTEPH are thought to be a misguided vascular remodelling process, which involves defective angiogenesis and delayed onset of fibrinolysis associated with endothelial dysfunction [5].

However, this historically accepted model for the pathogenesis of CTEPH has been subject to criticism, with those opposing it citing epidemiological data for PE and CTEPH, the demonstrable lack of some

Received: Feb 062015 | Accepted after revision: March 142015

Conflict of interest: Disclosures can be found alongside the online version of this article at err.ersjournals.com

Provenance: Publication of this peer-reviewed article was sponsored by Bayer Pharma AG, Berlin, Germany (principal sponsor, European Respiratory Review issue 136).

Copyright CERS 2015. ERR articles are open access and distributed under the terms of the Creative Commons Attribution Non-Commercial Licence 4.0. 
classic risk factors for VTE and the involvement of distal pulmonary artery disease in patients with CTEPH [6, 7]. Despite these past reservations, the thromboembolic basis of CTEPH is now well established [8].

\section{Definition and diagnostic criteria}

$\mathrm{PH}$ is defined as a resting mean pulmonary arterial pressure (mPAP) of $\geqslant 25 \mathrm{mmHg}$ measured by invasive right heart catheterisation $[1,9]$. There are a number of distinct types of $\mathrm{PH}$, which have been classified in terms of their aetiological, clinical and diagnostic features [1, 10]. This system of classification has undergone numerous revisions and clarifications, the most recent of which took place at the Fifth World Symposium on PH held in Nice, France, in 2013 [11]. Within the classification system, groups 1, 3, 4 and 5 comprise pre-capillary $\mathrm{PH}$, while post-capillary $\mathrm{PH}$, which is pulmonary venous hypertension due to left heart failure, falls into group 2 [1]. CTEPH represents group 4 and has been classified independently of all other forms of $\mathrm{PH}$, including group 1 pulmonary arterial hypertension (PAH), which can be difficult to distinguish from CTEPH clinically $[11,12]$.

A diagnosis of CTEPH can be made if, after 3 months of effective therapeutic anticoagulation, the patient's $\mathrm{mPAP}$ is $\geqslant 25 \mathrm{mmHg}$ and their pulmonary capillary wedge pressure is $\leqslant 15 \mathrm{mmHg}$, together with at least one mismatched segmental perfusion defect demonstrated by ventilation/perfusion $\left(V^{\prime} / Q^{\prime}\right)$ scanning, multidetector computed tomography angiography or pulmonary angiography [5]. $V^{\prime} / Q^{\prime}$ scanning is the preferred and recommended screening test in patients suspected of having CTEPH, with the main distinction between CTEPH and PAH being the segmental distribution of major vessel defects $[1,13]$. In contrast to patients with CTEPH, a $V^{\prime} / Q^{\prime}$ scan in a patient with PAH may appear normal, or show small peripheral unmatched and nonsegmental defects in perfusion [1]. A brief comparison of CTEPH and PAH is shown in table 1.

\section{Clinical features and treatment}

Early identification and accurate diagnosis of CTEPH is challenging as the condition may be completely asymptomatic during its initial development and progression $[2,14]$. This problem is further compounded by the clinical signs and symptoms of CTEPH being largely nonspecific [8]. As with other forms of $\mathrm{PH}$, patients with CTEPH will typically present with progressive exertional dyspnoea and reduced exercise tolerance, in addition to which they may also have fatigue, syncope and/or haemoptysis [15]. As CTEPH

TABLE 1 Comparison of chronic thromboembolic pulmonary hypertension (CTEPH) and pulmonary arterial hypertension (PAH) CTEPH PAH

Common pathophysiological link

Common symptoms

Aetiology

Risk factors

Disease phenotype

Diagnosis

Treatment

Microvascular arteriopathy resulting in endothelial dysfunction and vascular remodelling

Dyspnoea, fatigue, weakness and syncope

Unresolved emboli after PE

Unknown aetiology in the absence of previous $\mathrm{PE}$

Previous PE/VTE and recurrent VTE, infected pacemaker, ventriculoatrial shunt, splenectomy, antiphospholipid antibodies, and lupus anticoagulant

Primarily affects the elderly of both sexes, episodic course with "honeymoon" periods

Segmental perfusion defects in $V^{\prime} / Q^{\prime}$ scan, pulmonary angiography, CT

PEA surgery for suitable patients, balloon pulmonary angioplasty for segmental/ subsegmental disease, medical treatment targeting dysfunctional pathways in endothelial cells of inoperable patients
Abnormal proliferation of endothelium and smooth muscle in vessel walls, small vessel thrombi are possible

Genetic mutation, certain drugs/toxins, HIV, connective tissue disorders, congenital heart disease

Typically affects young women, progressive course

No segmental perfusion defects in $V^{\prime} / Q^{\prime}$ scan, right heart catheterisation

Medical treatment targeting dysfunctional pathways in endothelial cells

PE: pulmonary embolism; VTE: venous thromboembolism; $V^{\prime} / Q^{\prime}$ : ventilation/perfusion; CT: computed tomography; PEA: pulmonary endarterectomy. Information from [8]. 
progresses into the more advanced stages of the disease and right ventricular dysfunction becomes more pronounced, the clinical manifestations of right heart failure may become more obvious $[3,8]$.

Underdiagnosis and delay in the diagnosis of CTEPH are concerning because, unlike other types of $\mathrm{PH}$, CTEPH is potentially curable via surgical intervention in many patients [15]. Pulmonary endarterectomy (PEA) is a complex surgical procedure that involves the removal of the obstructive thromboembolic material from the patient's pulmonary vasculature $[16,17]$. This is possible in CTEPH, as opposed to other forms of $\mathrm{PH}$, because initially the condition is the result of occlusion predominantly occurring in the central and proximal pulmonary arteries $[2,8]$. However, a significant proportion of patients with CTEPH are unsuitable for PEA. This is most often due to involvement of the distal pulmonary vessels, the presence of significant comorbidities or the patient's refusal to undergo an operation [18]. For patients with CTEPH who are inoperable, or who have persistent/recurrent PH after PEA, the only currently approved pharmacological treatment is the soluble guanylate cyclase stimulator riociguat [13]. Clinical data on the use of pharmacological treatment in CTEPH are reviewed in this issue of the European Respiratory Review [19]. Without treatment the prognosis for patients with CTEPH is poor, with higher mortality rates associated with higher mPAPs, highlighting the need for timely diagnosis and early surgical intervention where possible [13, 20-22].

\section{Epidemiology}

CTEPH is understood to be a rare disease, with an epidemiology likely to be similar to that of PAH. There are significant obstacles to determining the overall incidence and prevalence of CTEPH accurately, and it is likely that they have been underestimated in the past [23]. Patients with CTEPH commonly lack an identifiable causative event due to the $\mathrm{PE}$ often being asymptomatic. In addition, initial symptoms may be nonspecific or absent. Other obstructing factors are referral bias and the possibility of CTEPH pre-existing in patients prior to $\mathrm{PE}[8,23,24]$.

Despite these inherent difficulties, the epidemiology of CTEPH has been an area of research interest, focused particularly on quantifying the rate of its occurrence following an acute PE. Early estimates suggested that CTEPH occurs in approximately $0.1-0.5 \%$ of patients who survive an episode of acute PE [2]. However, more recent prospective observational studies have reported the prevalence of CTEPH after acute PE as being between $0.4 \%$ and $9.1 \%$ [14, 25-32]. The wide range of estimates may have arisen due to differences in study design, patient populations or diagnostic methods and criteria, as well as a result of the obstacles mentioned above [32]. For example, some studies did not utilise right heart catheterisation to confirm the diagnosis of CTEPH $[3,14]$.

Previous arguments against the pathogenesis of CTEPH being thromboembolic in nature have cited the lack of a clearly documented history of either VTE or PE in approximately half of patients with CTEPH $[6,7]$. These concerns have now largely been addressed, with findings from an international prospective registry of patients with CTEPH reporting a history of acute PE in $74.8 \%$ of all patients with CTEPH and a history of deep venous thrombosis in $56.1 \%$ [18]. These data, considered in the context of a proportion of patients in whom PE and VTE occurs without clinical symptoms, emphasise the natural history of $\mathrm{CTEPH}$ as a potential long-term consequence of PE [2].

\section{Pathophysiology}

Understanding of the pathogenesis and subsequent progression of CTEPH has evolved within the conceptual framework of the condition as a dual pulmonary vascular disorder, with initial occlusion of proximal major vessels by nonresolution of a single or recurrent PE, which may trigger distal pulmonary arteriopathy and microvascular disease $[5,33,34]$. The aetiological factors that have been implicated are associated with aberrations in coagulation and thrombus resolution $[5,35,36]$. The most important risk factor for patients progressing from an acute PE to CTEPH is previous/recurrent PE or VTE. In a retrospective cohort study of cases from three European centres offering PEA, a comparison between patients with CTEPH and those with non-thromboembolic pre-capillary PAH found that a history of VTE and recurrent VTE were significantly more common in the CTEPH group than the PAH group (odds ratios of 4.5 and 14.5, respectively) [35]. A schematic overview of the pathophysiological concept of CTEPH is shown in figure 1 .

\section{Coagulation and thrombus resolution}

Despite the link between CTEPH and VTE, some classic plasmatic thromboembolic risk factors including antithrombin, protein $\mathrm{C}$ and protein $\mathrm{S}$ deficiency, and factor $\mathrm{V}$ Leiden mutation have been found not to be associated with CTEPH [5, 37]. However, elevated plasma concentrations of factor VIII, lupus anticoagulant and antiphospholipid antibodies, all three of which are risk factors for VTE, have been found to be associated with CTEPH $[35,37,38]$. Fibrinolytic factors have also been investigated, but have not been found to be significantly abnormal in patients with CTEPH [39]. Further nonplasmatic specific 


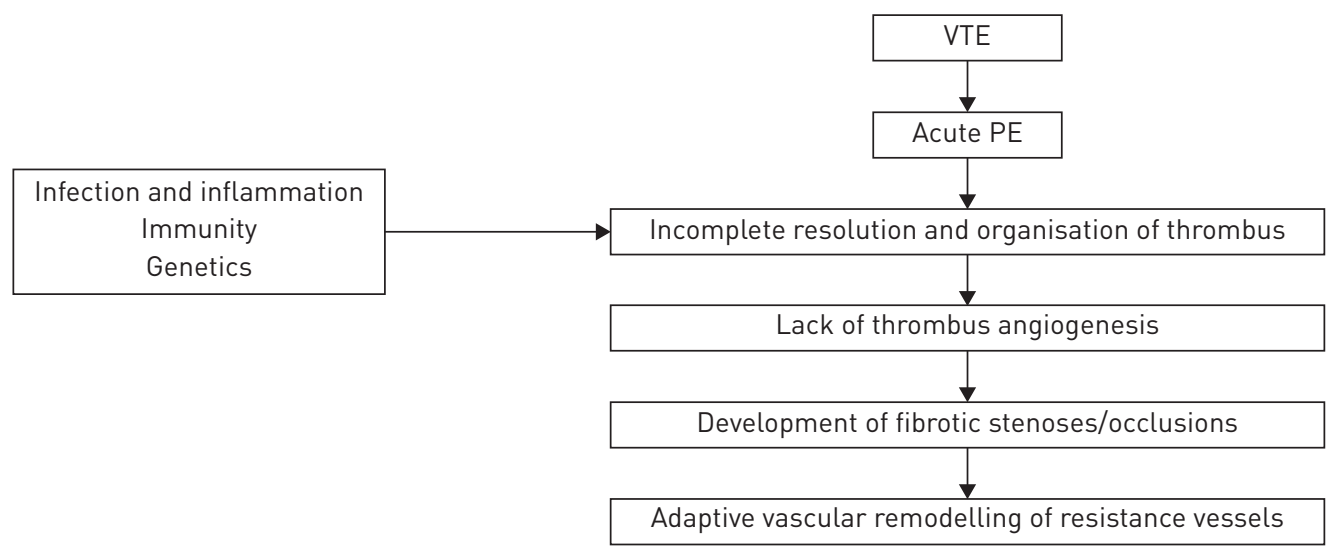

FIGURE 1 Schematic of the pathophysiological concept of chronic thromboembolic pulmonary hypertension following acute pulmonary embolism (PE). VTE: venous thromboembolism. Reproduced from [5] with permission from the publisher.

risk factors for VTE have also been identified as risk factors for CTEPH. Splenectomy, infected ventriculoatrial shunts, thyroid replacement therapy, malignancy and chronic inflammatory conditions, including osteomyelitis and inflammatory bowel disease, are all significantly associated with CTEPH and have a negative impact on prognosis [35, 40-42].

As with the other mechanisms involved in the pathogenesis of CTEPH, the causes of thrombus non-resolution have yet to be fully clarified. In otherwise healthy individuals, dissolution of large thrombi typically begins with initially rapid fibrinolysis followed by the formation of granulation tissue, similar to the process of wound healing, with a cellular response that leads to recruitment of leukocytes and endothelial progenitor cells with concomitant angiogenesis [43-46]. Initially, neutrophils are recruited into the resolving thrombus, where they promote ongoing fibrinolysis and collagenolysis. The neutrophils are followed by monocytes, which have a more expansive role involving the secretion or attraction of various chemokines, growth factors and proteases that promote thrombus reorganisation $[8,46]$. These normal mechanisms of thrombus breakdown may be altered by the presence of inflammation and associated inflammatory markers, such as C-reactive protein, monocyte chemotactic protein-1, tumour necrosis factor- $\alpha$ and interferon- $\gamma$-induced protein-10, which have been found to be upregulated in patients with CTEPH [47-50].

There have also been abnormal fibrinogen variants reported in CTEPH, which may be more resistant to cleavage and resolution $[36,51,52]$. These include a significant association between CTEPH and the fibrinogen A $\alpha$ polymorphism Thr312Ala, which has been shown to increase the resistance of fibrin to typical lytic processes [53, 54]. Taken together, these observations demonstrate a number of pathophysiological modalities that between them probably account for the non-resolution of thromboembolic material seen in $\mathrm{CTEPH}$, although further investigation is required.

Aberrations in angiogenesis during thrombus resolution have also been implicated in the pathogenesis of CTEPH. During normal angiogenesis, positive regulators of neovascularisation, such as vascular endothelial growth factor (VEGF) and fibroblast growth factor (FGF), are upregulated and cause endothelial activation [55]. VEGF and basic FGF are both found in organising thrombi, which suggests that they have a role in thrombus resolution and that augmenting the expression of these growth factors may enhance thrombus recanalisation [56]. Thromboembolic material removed from patients with CTEPH during PEA has been found to contain collagen-secreting cells. These cells may participate in the formation of a unique microenvironment within the organised thrombus that leads to dysfunctional endothelial cells, which do not support the process of angiogenesis [57, 58]. Downregulation of angiogenetic gene expression and a lack of functional endothelial cells in CTEPH thrombi may contribute to the failure of pulmonary vascular obstructions to resolve in these patients [5].

\section{Major vessel and microvascular disease}

The characteristic transformation of thromboembolic material, in the elastic pulmonary arteries, into organised fibrotic scar tissue with tight attachments to the medial layer, replacing the normal intima, can result not only in complete occlusion of the vessel lumen but also in the formation of varying grades of stenosis, webs and bands [1]. As a result of the persistent obstruction of affected arteries in CTEPH, pulmonary blood flow is redistributed to non-occluded vessels [3]. Redirection of cardiac output subjects these pulmonary vessels to elevated blood pressures and increased shear stress, which, in conjunction with the potential presence of inflammation and vasculopathic mediators, results in progressive arteriopathy in 
the distal arteries and arterioles [3, 8]. This arteriopathic process in CTEPH results in endothelial dysfunction and vascular remodelling of the pulmonary microvasculature, which resembles the mechanisms underlying PAH and leads to the formation of plexiform lesions in both conditions [15, 34, 59].

The small vessel vascular remodelling in CTEPH is characterised by thickening of all three layers of the vascular wall, and hypertrophy or hyperplasia of the predominant cell type of each layer (fibroblasts, smooth muscle cells and endothelial cells, respectively); it may also involve abnormal proliferation of pulmonary arterial smooth muscle cells and the accumulation of putative endothelial progenitor cells [57, 60-62]. These microvascular changes cause further worsening of the patient's $\mathrm{PH}$, and therefore reciprocal deterioration of their clinical condition, explaining the progressive nature of CTEPH even in the absence of recurrent thromboembolic events [3]. Concomitant small vessel arteriopathy also accounts for the documented lack of a linear correlation between measurable haemodynamic variables and the degree of vascular obstruction in $\mathrm{CTEPH}$, which distinguishes it from acute PE where this correlation does exist $[60,63]$.

\section{Conclusions}

CTEPH is a distinct form of $\mathrm{PH}$ both in terms of its aetiology and the way in which it responds to treatment; it is the only subset of $\mathrm{PH}$ which is amenable to curative surgical intervention. The pathophysiological processes underpinning CTEPH are complex and have not yet been fully elucidated. In time, further research will continue to clarify these mechanisms and may provide greater insight into the potential overlap between the pathogenesis of microvascular arteriopathy in CTEPH and in PAH. While existing epidemiological data have firmly established the thromboembolic nature of this condition, there is still a large margin of error between different studies, the result of which is that the exact prevalence and incidence of CTEPH remain unknown. Advances in these fields may allow mutual clinical improvements in the diagnosis and management of patients with CTEPH.

\section{Acknowledgements}

Editorial assistance was provided by Adelphi Communications Ltd (Bollington, UK), supported by Bayer Pharma AG.

\section{References}

1 Galiè N, Hoeper MM, Humbert M, et al. Guidelines for the diagnosis and treatment of pulmonary hypertension. Eur Respir J 2009; 34: 1219-1263.

2 Fedullo PF, Auger WR, Kerr KM, et al. Chronic thromboembolic pulmonary hypertension. N Engl J Med 2001; 345: $1465-1472$

3 Hoeper MM, Mayer E, Simonneau G, et al. Chronic thromboembolic pulmonary hypertension. Circulation 2006; 113: 2011-2020.

4 Kunieda T, Nakanishi N, Satoh T, et al. Prognoses of primary pulmonary hypertension and chronic major vessel thromboembolic pulmonary hypertension determined from cumulative survival curves. Intern Med 1999; 38 : 543-546.

5 Lang IM, Pesavento R, Bonderman D, et al. Risk factors and basic mechanisms of chronic thromboembolic pulmonary hypertension: a current understanding. Eur Respir J 2013; 41: 462-468.

6 Egermayer P, Peacock AJ. Is pulmonary embolism a common cause of chronic pulmonary hypertension? Limitations of the embolic hypothesis. Eur Respir J 2000; 15: 440-448.

7 Peacock A, Simonneau G, Rubin L. Controversies, uncertainties and future research on the treatment of chronic thromboembolic pulmonary hypertension. Proc Am Thorac Soc 2006; 3: 608-614.

8 Lang IM, Madani M. Update on chronic thromboembolic pulmonary hypertension. Circulation 2014; 130: 508-518.

9 Hatano S, Strasser T. Primary pulmonary hypertension: report on a WHO meeting, Geneva, 15-17 October 1973. Geneva, World Health Organization, 1975.

10 Simonneau G, Galiè N, Rubin LJ, et al. Clinical classification of pulmonary hypertension. J Am Coll Cardiol 2004; 43: Suppl. S, 5S-12S.

11 Simonneau G, Gatzoulis MA, Adatia I, et al. Updated clinical classification of pulmonary hypertension. J Am Coll Cardiol 2013; 62: Suppl., D34-D41.

12 Pepke-Zaba J, Jansa P, Kim NH, et al. Chronic thromboembolic pulmonary hypertension: role of medical therapy. Eur Respir J 2013; 41: 985-990.

13 Kim NH, Delcroix M, Jenkins DP, et al. Chronic thromboembolic pulmonary hypertension. J Am Coll Cardiol 2013; 62: Suppl., D92-D99.

14 Pengo $\mathrm{V}$, Lensing AW, Prins $\mathrm{MH}$, et al. Incidence of chronic thromboembolic pulmonary hypertension after pulmonary embolism. N Engl J Med 2004; 350: 2257-2264.

15 Hoeper MM, Madani MM, Nakanishi N, et al. Chronic thromboembolic pulmonary hypertension. Lancet Respir Med 2014; 2: 573-582.

16 Moser KM, Auger WR, Fedullo PF, et al. Chronic thromboembolic pulmonary hypertension: clinical picture and surgical treatment. Eur Respir J 1992; 5: 334-342.

17 Jenkins D. Pulmonary endarterectomy: the potentially curative treatment for patients with chronic thromboembolic pulmonary hypertension. Eur Respir Rev 2015; 24: 263-271.

18 Pepke-Zaba J, Delcroix M, Lang I, et al. Chronic thromboembolic pulmonary hypertension (CTEPH): results from an international prospective registry. Circulation 2011; 124: 1973-1981.

19 Hoeper MM. Pharmacological therapy for patients with chronic thromboembolic pulmonary hypertension. Eur Respir Rev 2015; 24: 272-282. 
20 Lewczuk J, Piszko P, Jagas J, et al. Prognostic factors in medically treated patients with chronic pulmonary embolism. Chest 2001; 119: 818-823.

21 Riedel M, Stanek V, Widimsky J, et al. Longterm follow-up of patients with pulmonary thromboembolism. Late prognosis and evolution of hemodynamic and respiratory data. Chest 1982; 81: 151-158.

22 Mayer E, Jenkins D, Lindner J, et al. Surgical management and outcome of patients with chronic thromboembolic pulmonary hypertension: results from an international prospective registry. J Thorac Cardiovasc Surg 2011; 141: 702-710.

23 Lang IM. Chronic thromboembolic pulmonary hypertension - not so rare after all. N Engl J Med 2004; 350: 2236-2238.

24 Tapson VF, Humbert M. Incidence and prevalence of chronic thromboembolic pulmonary hypertension: from acute to chronic pulmonary embolism. Proc Am Thorac Soc 2006; 3: 564-567.

25 Becattini C, Agnelli G, Pesavento R, et al. Incidence of chronic thromboembolic pulmonary hypertension after a first episode of pulmonary embolism. Chest 2006; 130: 172-175.

26 Miniati M, Monti S, Bottai M, et al. Survival and restoration of pulmonary perfusion in a long-term follow-up of patients after acute pulmonary embolism. Medicine (Baltimore) 2006; 85: 253-262.

27 Dentali F, Donadini M, Gianni M, et al. Incidence of chronic pulmonary hypertension in patients with previous pulmonary embolism. Thromb Res 2009; 124: 256-258.

28 Martí D, Gómez V, Escobar C, et al. Incidencia de hipertension pulmonar tromboembolica cronica sintomatica y asintomatica [Incidence of symptomatic and asymptomatic chronic thromboembolic pulmonary hypertension]. Arch Bronconeumol 2010; 46: 628-633.

29 Poli D, Grifoni E, Antonucci E, et al. Incidence of recurrent venous thromboembolism and of chronic thromboembolic pulmonary hypertension in patients after a first episode of pulmonary embolism. J Thromb Thrombolysis 2010; 30: 294-299.

30 Surie S, Gibson NS, Gerdes VE, et al. Active search for chronic thromboembolic pulmonary hypertension does not appear indicated after acute pulmonary embolism. Thromb Res 2010; 125: e202-e205.

31 Korkmaz A, Ozlu T, Ozsu S, et al. Long-term outcomes in acute pulmonary thromboembolism: the incidence of chronic thromboembolic pulmonary hypertension and associated risk factors. Clin Appl Thromb Hemost 2012; 18: 281-288.

32 Guérin L, Couturaud F, Parent F, et al. Prevalence of chronic thromboembolic pulmonary hypertension after acute pulmonary embolism. Prevalence of CTEPH after pulmonary embolism. Thromb Haemost 2014; 112: 598-605.

33 Moser KM, Auger WR, Fedullo PF. Chronic major-vessel thromboembolic pulmonary hypertension. Circulation 1990; 81: 1735-1743.

34 Moser KM, Bloor CM. Pulmonary vascular lesions occurring in patients with chronic major vessel thromboembolic pulmonary hypertension. Chest 1993; 103: 685-692.

35 Bonderman D, Wilkens H, Wakounig S, et al. Risk factors for chronic thromboembolic pulmonary hypertension. Eur Respir J 2009; 33: 325-331.

36 Morris TA, Marsh JJ, Chiles PG, et al. High prevalence of dysfibrinogenemia among patients with chronic thromboembolic pulmonary hypertension. Blood 2009; 114: 1929-1936.

37 Wolf M, Boyer-Neumann C, Parent F, et al. Thrombotic risk factors in pulmonary hypertension. Eur Respir J 2000; 15: 395-399.

38 Wong CL, Szydlo R, Gibbs S, et al. Hereditary and acquired thrombotic risk factors for chronic thromboembolic pulmonary hypertension. Blood Coagul Fibrinolysis 2010; 21: 201-206.

39 Olman MA, Marsh JJ, Lang IM, et al. Endogenous fibrinolytic system in chronic large-vessel thromboembolic pulmonary hypertension. Circulation 1992; 86: 1241-1248.

40 Bonderman D, Skoro-Sajer N, Jakowitsch J, et al. Predictors of outcome in chronic thromboembolic pulmonary hypertension. Circulation 2007; 115: 2153-2158.

41 Bonderman D, Jakowitsch J, Adlbrecht C, et al. Medical conditions increasing the risk of chronic thromboembolic pulmonary hypertension. Thromb Haemost 2005; 93: 512-516.

42 Jaïs X, Ioos V, Jardim C, et al. Splenectomy and chronic thromboembolic pulmonary hypertension. Thorax 2005; 60: $1031-1034$

43 Modarai B, Humphries J, Burnand KG, et al. Adenovirus-mediated VEGF gene therapy enhances venous thrombus recanalization and resolution. Arterioscler Thromb Vasc Biol 2008; 28: 1753-1759.

44 Waltham M, Burnand KG, Collins M, et al. Vascular endothelial growth factor enhances venous thrombus recanalisation and organisation. Thromb Haemost 2003; 89: 169-176.

45 Modarai B, Burnand KG, Sawyer B, et al. Endothelial progenitor cells are recruited into resolving venous thrombi. Circulation 2005; 111: 2645-2653.

46 Wakefield TW, Myers DD, Henke PK. Mechanisms of venous thrombosis and resolution. Arterioscler Thromb Vasc Biol 2008; 28: 387-391.

47 Zabini D, Heinemann A, Foris V, et al. Comprehensive analysis of inflammatory markers in chronic thromboembolic pulmonary hypertension patients. Eur Respir J 2014; 44: 951-962.

48 Kimura H, Okada O, Tanabe N, et al. Plasma monocyte chemoattractant protein-1 and pulmonary vascular resistance in chronic thromboembolic pulmonary hypertension. Am J Respir Crit Care Med 2001; 164: 319-324.

49 Quarck R, Nawrot T, Meyns B, et al. C-reactive protein: a new predictor of adverse outcome in pulmonary arterial hypertension. I Am Coll Cardiol 2009; 53: 1211-1218.

50 Langer F, Schramm R, Bauer M, et al. Cytokine response to pulmonary thromboendarterectomy. Chest 2004; 126: 135-141.

51 Miniati M, Fiorillo C, Becatti M, et al. Fibrin resistance to lysis in patients with pulmonary hypertension other than thromboembolic. Am J Respir Crit Care Med 2010; 181: 992-996.

52 Morris TA, Marsh JJ, Chiles PG, et al. Fibrin derived from patients with chronic thromboembolic pulmonary hypertension is resistant to lysis. Am J Respir Crit Care Med 2006; 173: 1270-1275.

53 Suntharalingam J, Goldsmith K, van Marion V, et al. Fibrinogen A $\alpha$ Thr312Ala polymorphism is associated with chronic thromboembolic pulmonary hypertension. Eur Respir J 2008; 31: 736-741.

54 Li JF, Lin Y, Yang YH, et al. Fibrinogen A $\alpha$ Thr312Ala polymorphism specifically contributes to chronic thromboembolic pulmonary hypertension by increasing fibrin resistance. PLoS One 2013; 8: e69635. 
Distler JH, Hirth A, Kurowska-Stolarska M, et al. Angiogenic and angiostatic factors in the molecular control of angiogenesis. Q J Nucl Med 2003; 47: 149-161.

56 Waltham M, Burnand KG, Collins M, et al. Vascular endothelial growth factor and basic fibroblast growth factor are found in resolving venous thrombi. J Vasc Surg 2000; 32: 988-996.

57 Sakao S, Hao H, Tanabe N, et al. Endothelial-like cells in chronic thromboembolic pulmonary hypertension: crosstalk with myofibroblast-like cells. Respir Res 2011; 12: 109.

58 Firth AL, Yao W, Ogawa A, et al. Multipotent mesenchymal progenitor cells are present in endarterectomized tissues from patients with chronic thromboembolic pulmonary hypertension. Am J Physiol Cell Physiol 2010; 298: C1217-C1225.

59 Yi ES, Kim H, Ahn H, et al. Distribution of obstructive intimal lesions and their cellular phenotypes in chronic pulmonary hypertension. A morphometric and immunohistochemical study. Am J Respir Crit Care Med 2000; 162: $1577-1586$

60 Galiè N, Kim NH. Pulmonary microvascular disease in chronic thromboembolic pulmonary hypertension. Proc Am Thorac Soc 2006; 3: 571-576.

61 Ogawa A, Firth AL, Yao W, et al. Inhibition of mTOR attenuates store-operated Ca2 ${ }^{+}$entry in cells from endarterectomized tissues of patients with chronic thromboembolic pulmonary hypertension. Am J Physiol Lung Cell Mol Physiol 2009; 297: L666-L676.

62 Wynants M, Quarck R, Ronisz A, et al. Effects of C-reactive protein on human pulmonary vascular cells in chronic thromboembolic pulmonary hypertension. Eur Respir J 2012; 40: 886-894.

63 Azarian R, Wartski M, Collignon MA, et al. Lung perfusion scans and hemodynamics in acute and chronic pulmonary embolism. J Nucl Med 1997; 38: 980-983. 